\title{
Cuidados ao se Extrair Dimensões de Fotografia/Filmagem em Soldagem
}

\author{
(Issues before Performing Measurements from Pictures/Films in Welding)
}

\author{
Louriel O. Vilarinho ${ }^{1}$, Bill Lucas ${ }^{2}$, Sayee Raghunathan ${ }^{2}$ \\ ${ }^{1}$ Universidade Federal de Uberlândia, Laprosolda - Centro para Pesquisa e Desenvolvimento de Processos de Soldagem, Uberlândial \\ MG,38400-902, vilarinho@mecanica.ufu.br \\ ${ }^{2}$ The Welding Institute - TWI, Granta Park, Great Abington, Cambridge CB1 6AL, United Kingdom, bill.lucas@twi.co.uke Sayee. \\ Raghunathan@twi.co.uk
}

\begin{abstract}
Resumo
As técnicas de fotografia e filmagem abriram caminhos para estudos em todas as áreas da soldagem e têm sido extensivamente utilizadas para se avaliar características tais como microconstituintes, fases, estruturas, transferência metálica, escoamento de gases, macrografias, deformações e medições geométricas de peças. Embora haja este uso fundamental na soldagem, tem-se notado constantes erros na utilização das técnicas de fotografia e filmagem, como erros de posicionamento, calibração, escolha do sensor, tipo e compactação de imagem. Objetiva-se assim trazer à discussão os critérios e cuidadosos necessários durante medições realizadas a partir de fotografias/ filmagens em soldagem. São abordados ao longo do texto os principais detalhes relacionados ao tema de forma prática para que o usuário possa aproveitar todo o potencial de sua fotografia/filmagem. Espera-se a discussão aqui apresentada contribua para o melhor aproveitamento e correto uso das imagens/filmes obtidos em processos de soldagem. Espera-se também que haja comentários sobre o texto no sentido de melhorar e trazer à tona esta importante discussão no âmbito da Revista Soldagem \& Inspeção.
\end{abstract}

Palavras-chave: Metrologia; Sistema de Visão; Compensação.

\begin{abstract}
Photographic and filming techniques have opened different study fields in all welding area and have been extensively employed to assess characteristics such as micro-constituents, phases, structures, metal transfer, gas flow, macro-etches, distortion and geometric measurements in workpieces. Despite this fundamental use in welding, constant mistakes and misuses of these techniques have been noticed, as positioning error, calibration, sensor selection, and type of image. Therefore, it is aimed here to address criteria required during measurements from pictures/films in welding. The main details are discussed in a practical form, so the user can benefit from the full potential of the technique. It is expected that the presented discussion contributes to the better and correct use of pictures/films from different welding processes. The authors also expect comments and suggestions to improve and bring this fundamental and important issue to the spotlight of the Revista Soldagem \& Inspeção.
\end{abstract}

Key-words: Metrology; Vision System; Compensation.

\section{Introdução}

Não há dúvidas com relação ao potencial investigativo de uma imagem/filmagem. As técnicas de fotografia e filmagem abriram caminhos para estudos em todas as áreas da soldagem, seja metalurgia, processos, fabricação ou projeto. Microconstituintes, estruturas, transferência metálica, escoamento de gases, macrografias, deformações, medições geométricas de peças, dentre outras características têm sido estudadas com tais técnicas.

Embora tenham uso fundamental na soldagem, tem-se notado constantes erros na utilização das técnicas de fotografia e

(Recebido em 17/03/2009; Texto Final em 01/12/2009). filmagem, como erros de posicionamento, calibração, escolha do sensor, tipo e compactação de imagem. Desta forma, pretende-se abordar os principais detalhes para se extrair dimensões de uma imagem de forma prática para que o usuário possa aproveitar todo o potencial de sua fotografia/filmagem. Além disto, esperase que haja comentários sobre o texto no sentido de melhorar e trazer à tona esta importante discussão. Ressalta-se a finalidade do texto é apresentar um desafio, onde muitos usam a técnica, mas não a empregam de forma otimizada.

Os tópicos seguintes procuram abordar os principais cuidados ao se medir imagens e filmes a partir da soldagem, onde são cobertos os temas de compensações geométricas para o posicionamento da câmera e a otimização de seu posicionamento, informações referentes à imagem e algumas características de câmera e sensores. 


\section{Cuidados Principais}

Como exemplo prático, a Figura 1 traz a necessidade de se filmar a poça de soldagem. A forma mais direta de isto ser feito é através do uso de uma câmera protegida por uma lente utilizada em máscara de soldagem. A luz para observação do fenômeno provém do arco elétrico e a intensidade da visualização pode ser regulada através do grau do filtro utilizado, assim como um soldador o faria em função da corrente, comprimento de arco, processo, material, etc. Esta figura mostra a câmera colocada em um ângulo de $45^{\circ}$. Este valor será discutido mais adiante.

Neste tipo de montagem, onde se utiliza uma lente de soldagem, o detalhe principal é que o foco deve ser encontrado após a soldagem ser iniciada. Entretanto, um ajuste grosseiro do foco deve ser realizado antes da soldagem, sem a presença do filtro (lente de soldagem). Perde-se o foco da imagem devido à difração dos raios de luz no filtro, que é um dos preceitos utilizados no desenvolvimento de produtos voltados para a segurança do trabalho em soldagem [1].

Caso esteja se realizando filmagem, deve-se atentar para a quantidade de quadros por segundo necessária. Os fenômenos térmicos demandam tempo para acontecer e podem ser estimados pelo Número de Fourier [2]. De uma forma prática, caso o usuário esteja utilizando a filmagem como um sistema seguidor de junta, uma taxa de 10 quadros por segundo (qps) é suficiente. Para um estudo dimensional da poça recomenda-se 25 qps. Por outro lado, caso o usuário pretenda observar movimentação da poça e seus elementos (escória, por exemplo), uma taxa mínima de 500 qps se faz necessária [3, 4]. Deve-se ressaltar que, normalmente, à medida que se aumenta a taxa de quadros, reduz-se o tempo de exposição ('shutter') de cada um deles, ou seja, a quantidade de luz que entra. Isto deve ser levando em conta na escolha do filtro/lente. Vale lembrar também que os sistemas mais flexíveis de filmagem permitem ajustar o tempo de exposição de cada quadro independentemente da sua taxa, dentro de uma faixa operacional. Neste sentido, é possível não se utilizar a lente/ filtro, balanceando toda a luz recebida pelo sistema apenas com a correta exposição do mesmo [5]. A distância focal e a abertura do diafragma também influenciam na quantidade de luz que entra, sendo discutidas mais a frente.

Uma segunda abordagem para se atingir o objetivo de se visualizar a poça é mostrada na Figura 2, sendo utilizada em seguidores de junta e para a visualização do contorno da poça de soldagem. Aqui uma luz auxiliar é utilizada. Esta fonte de luz deve possuir intensidade suficiente para superar a radiação luminosa do arco, o que não é uma tarefa fácil. Assim, é mais interessante utilizar uma fonte de luz que tenha comprimento de onda numa faixa onde o arco elétrico emita menos radiação. Historicamente na soldagem [3, 6-9], diferentes comprimentos de onda foram testados e aqueles que proporcionaram os melhores resultados foram o $633 \mathrm{~nm}$ (a partir de laser vermelho de He-Ne) e o 904 $\mathrm{nm}$ (a partir de diodos laser emitindo no infravermelho). Ao se utilizar tais comprimentos de onda deve-se ter em mente dois fatos cruciais: a câmera deve ter sensibilidade no comprimento de onda em uso e que se utilize um filtro óptico que deixe passar uma pequena faixa de radiação em torno do comprimento de onda (normalmente esta faixa é de $1 \mathrm{~nm}$ ). Como este filtro deixa passar apenas uma pequena faixa (ou banda) do espectro de radiação, ele é chamado de filtro óptico passa-banda.

Deve-se ressaltar nesta segunda abordagem que não necessariamente a câmera e o feixe precisam estar alinhados. $\mathrm{O}$ alinhamento é benéfico, pois requer menos potência luminosa da fonte de luz, dada a reflexão especular da superfície. Por outro lado, não se deve esquecer que há reflexão difusa, ou seja, em todas as direções e, portanto, a câmera poderia ser colocada em qualquer posição, o que se traduz em flexibilidade na montagem do sistema, principalmente quando há restrições de espaço. Numa versão extremamente compacta, a fonte de luz pode estar coaxialmente à câmera, ao redor desta. Isto facilita a acessibilidade do sistema, mas requer uma fonte luminosa de muito mais alta potência [3]. Para tanto, diodos laser têm sido empregados, com fonte de potência pulsada com pico de potência de $90 \mathrm{~W}$. Uma ressalva muito importante deve ser feita aqui. A classificação do laser a ser utilizado é primordial para sua operacionalidade do ponto de vista de segurança do trabalho. Os lasers He-Ne normalmente utilizados em soldagem são contínuos com potência da ordem de $25 \mathrm{~mW}$ e recebem classificação Classe II, ou seja, não é necessário uso constante de máscara e somente a exposicão direta aos olhos deve ser evitada. Já os laser diodos utilizados de forma pulsada são Classe IIIB, ou seja, o operador que se encontra próximo a ele deve usar óculos, embora a reflexão difusa do mesmo não seja considerada um problema [10].

Em relação ao deslocamento do sistema de visão, uma vez que o movimento é relativo entre o eletrodo e a peça no caso da soldagem a arco, para se facilitar o estudo, pode-se fixar o eletrodo e movimentar a peça. Entretanto, esta não é a opção principal na prática. Assim, o cabeamento deve ser levado em consideração. Uma tendência recente é o uso de sistemas de comunicação sem fio [11], o que parece muito promissor, mas precisa ser melhor desenvolvido para ter robustez no ambiente da soldagem.

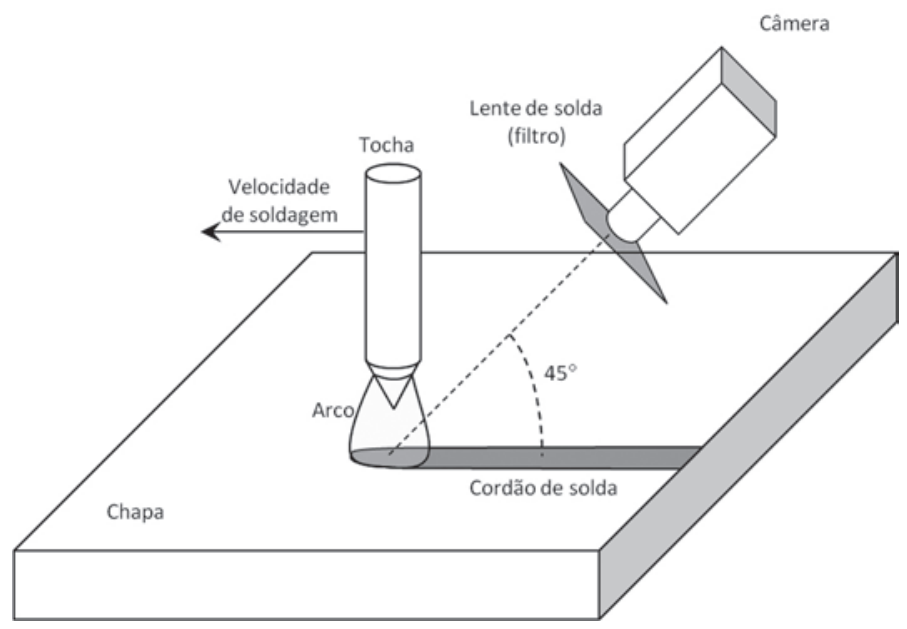

Figura 1. Primeira abordagem para visualização da poça, onde o arco é a própria fonte de luz [16].

Destas duas abordagens apresentadas, diferentes observações da imagem especular da poça são reportadas [3, 4], como por 
exemplo, o estudo da movimentação de escória e vórtices [12] e termometria [13], inclusive com sofisticações como uso de grade de laser $[14,15]$ para caracterização tridimensional da poça.

\section{1. Ângulo de Posicionamento e Visão}

Para exemplificar o uso em soldagem, tomar-se-á a Figura 3. Entretanto, apesar dos exemplos aqui apresentados estão ligados ao estudo do arco e da poça de fusão, os seus princípios são gerais e de aplicação em basicamente qualquer área do conhecimento. Ao se iniciar a montagem para visualização, um dos primeiros questionamentos é com relação ao posicionamento da câmera e da fonte de luz, caso exista. Num primeiro momento é intuitivo colocar a câmera a $45^{\circ}$ em relação ao plano da peça, sendo matematicamente correto fazê-lo, como será mostrado adiante. Entretanto, há a tendência de tentar visualizar o máximo possível dos elementos de uma soldagem (eletrodo, arco, poça, chapa e bocal). Assim, pode-se passar a trabalhar com ângulos de posicionamento mais raso em relação à peça (Figura 3), para que não seja necessário aumentar a altura da tocha e sair das condições operacionais normalmente utilizadas em soldagem.

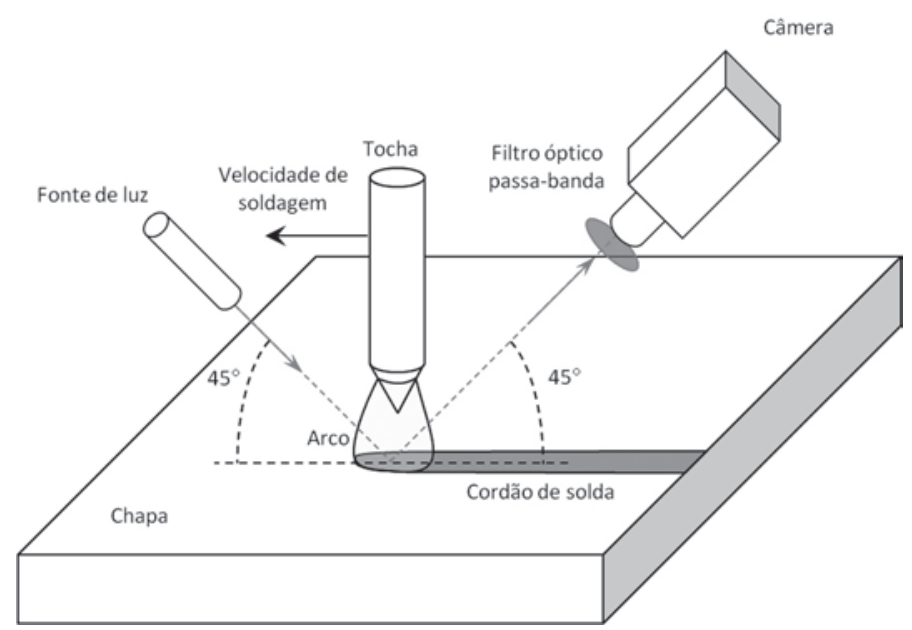

Figura 2. Segunda abordagem para visualização da poça, onde há uma fonte de luz externa e filtro óptico [16].

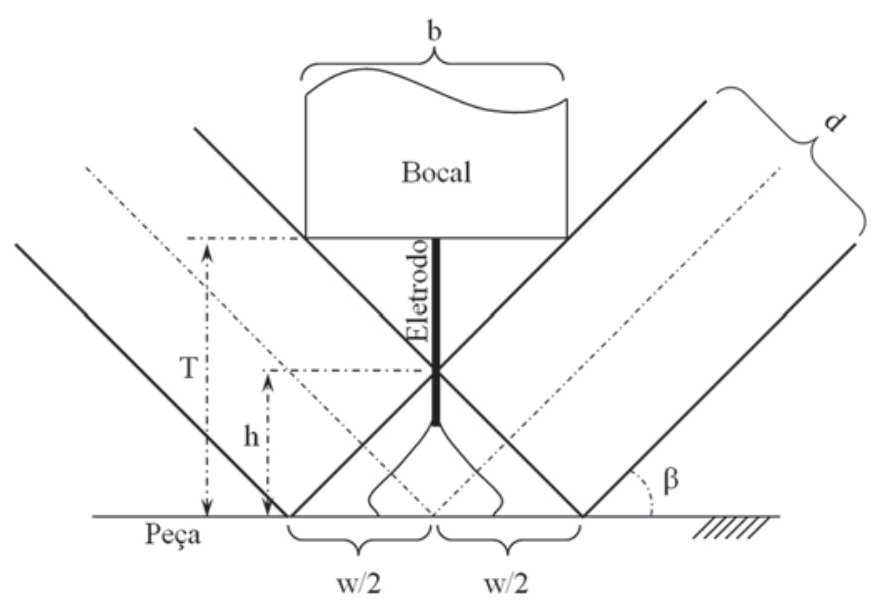

Figura 3. Visão esquemática da contra-iluminação com respectivas dimensões.
Dada as infinitas possibilidades de se trabalhar com os valores geométricos da Figura 3, deve-se buscar aquelas mais apropriadas. Para tanto, estabelece-se o modelo matemático das Equações 1 a 3, com uso de simples relações geométricas obtidas a partir da Figura 3. Ressalta-se que as imagens apresentadas que não contém citação bibliográfica foram derivadas da experiência dos autores. Na pesquisa bibliográfica, não foi encontrado na literatura o equacionamento/metodologia seguintes e, portanto, considera-se inédita.

$$
\begin{aligned}
& h=d \operatorname{sen} \beta \\
& w=2 d \cos \beta \\
& h=\frac{T \cdot w}{w+b}
\end{aligned}
$$

onde, h é a máxima altura iluminada (notar que $\mathrm{h}$ não é a altura da tocha/bocal, ou seja, é preciso trabalhar na relação entre comprimento de eletrodo e altura da tocha);

w é a largura iluminada na chapa (caso o feixe seja circular, w é o diâmetro iluminado);

d é o diâmetro do feixe de luz incidente originado na fonte de luz;

$\beta$ é o ângulo de incidência do feixe (neste caso igual ao ângulo de visão do observador;

$\mathrm{T}$ é a distância tocha-peça;

b é o diâmetro do bocal;

Este equacionamento consegue estabelecer os limites operacionais da visualização, conforme mostrado na Figura 4. Embora esta figura demonstre a tendência em se escolher um ou outro valor para o diâmetro do feixe e ângulo de incidência , ela não apresenta o melhor ponto operacional, ou seja, onde se consegue obter uma imagem com maior campo de visão. Além disto, este ponto operacional é função das dimensões operacionais da soldagem (altura da tocha e diâmetro do bocal), como mostra a Figura 5, obtida a partir da Equação 4.

$$
d=\frac{2 T-b \cdot \operatorname{tg} \beta}{2 \operatorname{sen} \beta}
$$

A princípio, o objetivo de uma visualização é conseguir observar o máximo possível de objetos em um dado quadro/ imagem, ou seja, é necessário maximizar os valores de $\mathrm{h}$ e w. Entretanto, há limites para o valor de $\mathrm{T}$ e b. Considerando que normalmente a distância tocha-peça varie entre 10 e $30 \mathrm{~mm}$ e assumindo um diâmetro de bocal de $25 \mathrm{~mm}$, é possível otimizar os valores do diâmetro do feixe de luz e do ângulo de visão para se maximizar as dimensões a serem visualizadas h e w. De antemão, sabe-se que quanto maio o diâmetro do feixe, maior será a área a ser visualizada. Este pré-conhecimento pode ser utilizado para verificação dos resultados da otimização. Mas primeiramente, deve-se estabelecer os limites da otimização das Equações 1 a 3, estabelecendo as seguintes desigualdades, a partir dos valores admitidos: 

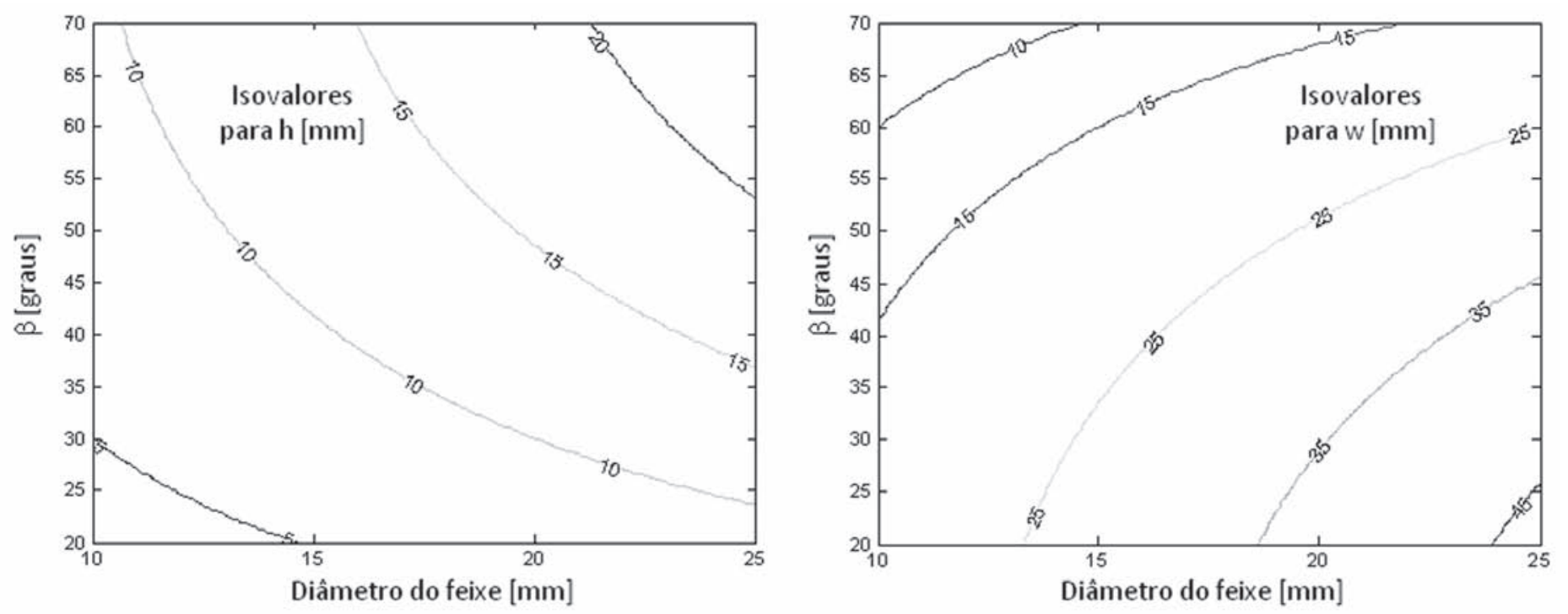

Figura 4. Isovalores para a máxima altura iluminada da tocha (h) e da dimensão iluminada da chapa (w) em função do diâmetro do feixe e ângulo $\beta$.

$$
\begin{aligned}
& \frac{10 \cdot w}{w+25}-h \leq 0 \\
& h-\frac{30 \cdot w}{w+25} \leq 0
\end{aligned}
$$

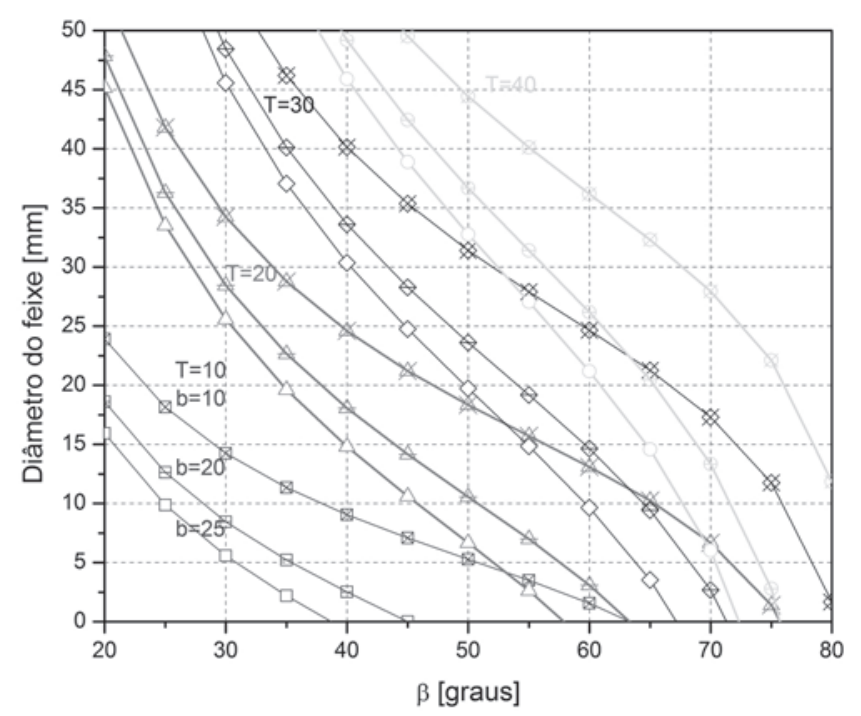

Figura 5. Relação entre diâmetro do feixe e ângulo $\beta$ para diferentes valores de altura da tocha $(\mathrm{T})$ e diâmetro de bocal (b), onde: símbolo quadrado $(\mathrm{T}=10 \mathrm{~mm})$; símbolo triângulo $(\mathrm{T}=20)$; símbolo losango $(\mathrm{T}=30)$; símbolo círculo $(\mathrm{T}=40)$; símbolo vazio $(\mathrm{b}=25 \mathrm{~mm})$; símbolo com traço horizontal $(\mathrm{b}=20 \mathrm{~mm})$ e símbolo com cruz $(\mathrm{b}=10 \mathrm{~mm})$.

Utilizando-se um algoritmo de otimização [16], calcula-se que para um diâmetro máximo de feixe de $25 \mathrm{~mm}$ (limite superior da variável d), os valores a serem utilizados são $d=25 \mathrm{~mm} ; \beta=$ $44,8^{\circ} ; \mathrm{h}=17,6 \mathrm{~mm}$ e $\mathrm{w}=35,5 \mathrm{~mm}$, para se obter a maior área de visualização pssível. Este resultado poderia induzir à conclusão que deve-se sempre usar um ângulo de $45^{\circ}$ para os sistema de visão. Entretanto, ao se alterar o diâmetro máximo possível do feixe (em função da disponibilidade para o usuário), altera-se o ponto operacional. Por exemplo, para um diâmetro máximo de feixe igual a $50 \mathrm{~mm}$, obtém-se $\mathrm{d}=50 \mathrm{~mm} ; \beta=27,8^{\circ} ; \mathrm{h}=23,4$ $\mathrm{mm}$ e $\mathrm{w}=88,4 \mathrm{~mm}$.

Esta alteração de ponto operacional é mais acentuada para menores valores de $\mathrm{T}$ (altura da tocha) e valores extremos de b (diâmetro do bocal), como mostra a Figura 6. Esta figura foi obtida pela otimização ponto-a-ponto de cada combinação das geometrias apresentadas na Figura 3 e discutidas até aqui, tendo como um diâmetro máximo de feixe $25 \mathrm{~mm}$.

Embora tenham sido demonstrados os melhores valores para o ângulo de incidência $\beta$, deve-se atentar para os cálculos matemáticos de todos os ângulos envolvidos na visualização. Em outras palavras, deve-se ter muito cuidado ao se extrair medidas das imagens, para que estas tenham o valor correto e não sejam uma componente da medida verdadeira. É intuitivo pensar que se uma fotografia for tirada de forma perpendicularmente perfeita em relação à normal do plano do objeto a ser visualizado, não há necessidade de se realizar correções geométricas devido ao posicionamento da câmera (entretanto, pode-se demandar correções devido a aberrações/distorções ópticas das lentes utilizadas). Por outro lado, se a imagem for tirada 'olhandose' de lado para o objeto, o que será observado é a projeção do objeto no plano da lente da câmera/observador. Assim, a Figura 7a traz a câmera posicionada no Ponto A e pretende-se tirar uma foto do cordão de solda (objeto) e medir sua largura w. 

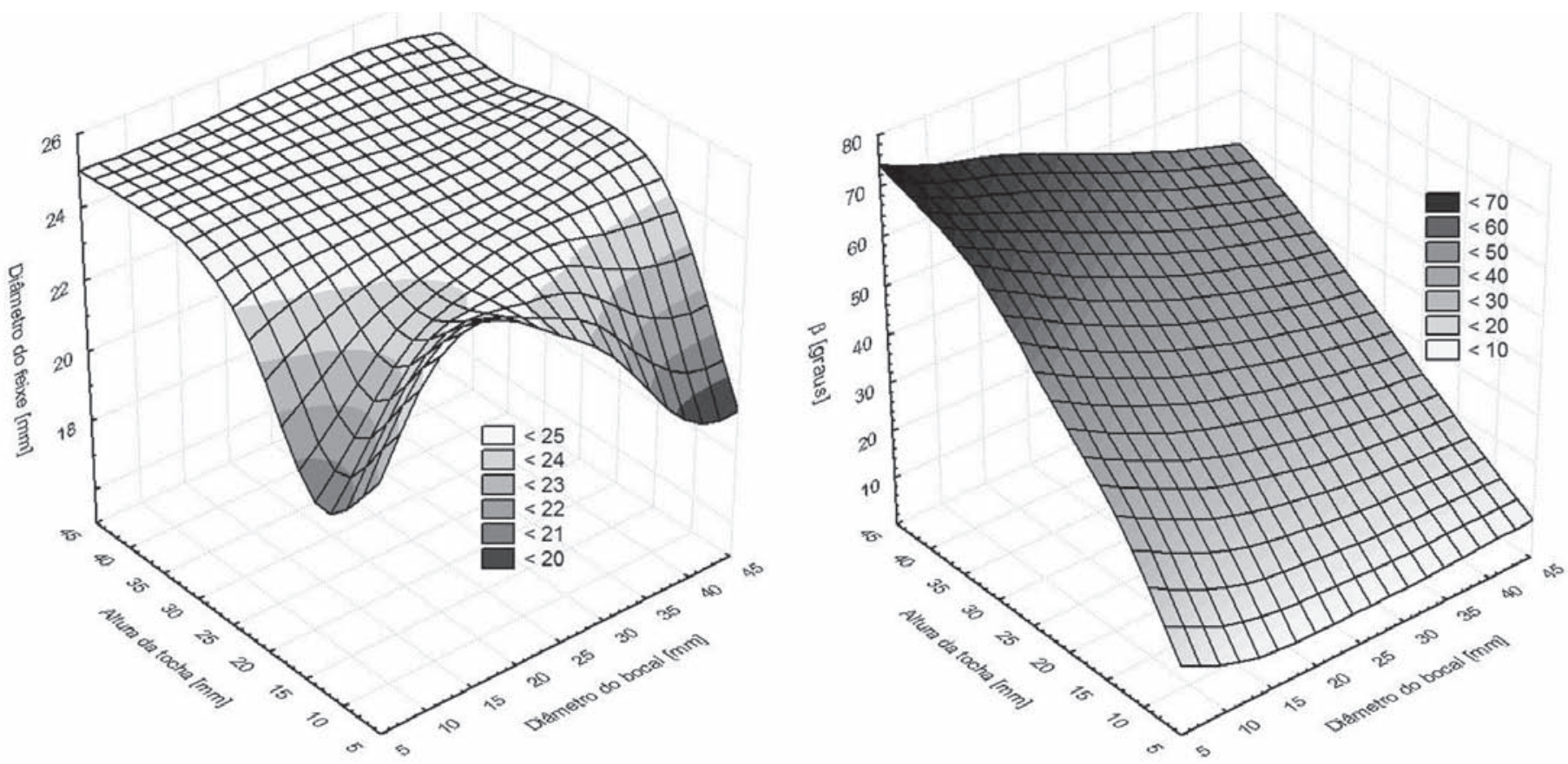

Figura 6. Valores otimizados para o diâmetro do feixe e do ângulo $\beta$ para diferentes valores de altura de tocha e diâmetro de bocal.
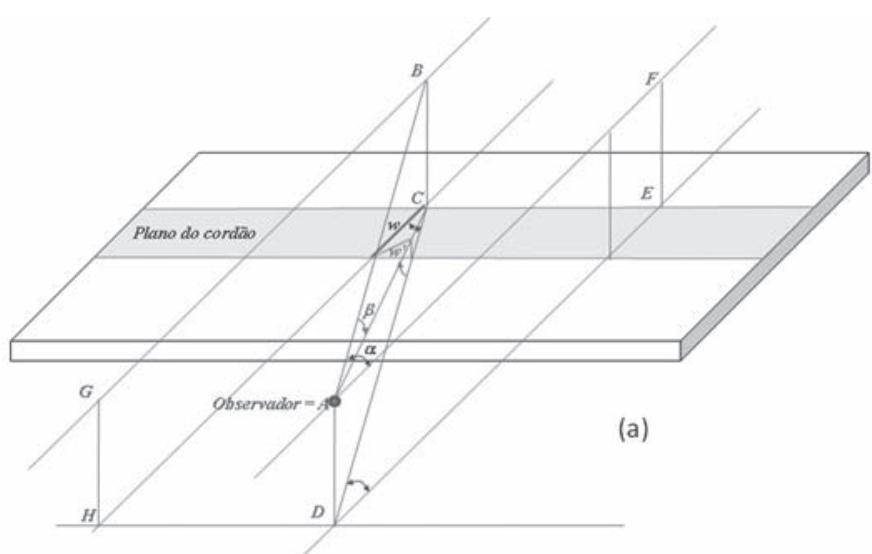

(a)

modelos, a serem apresentados matematicamente a seguir, são modelos simplificados e que um sistema de rotação e translação de coordenadas com base matricial é o modelo mais completo para a situação. Assim, fica aqui registrado, como é um desafio tecnológico, para os interessados em desenvolver o tema de transformações matemáticas em medidas tridimensionais nãolineares para objetos de soldagem.

Para o primeiro modelo, construindo-se as linhas auxiliares mostradas na Figura 7b, é possível demonstrar que:

$$
w^{\prime}=\frac{w}{\cos \alpha} \sqrt{\operatorname{tg}^{2} \beta+\operatorname{sen}^{2} \alpha}
$$

onde, w é a largura real do cordão;

w' é a projeção da largura w do cordão na direção do observador, i.e., a dimensão medida pelo sistema de visão;

$\alpha$ é o ângulo de rotação do plano AFED em torno de AD, i.e., $\alpha$ é o ângulo entre $\mathrm{AF}$ e $\mathrm{AB}$, que é igual ao ângulo entre $\mathrm{DE}$ e DC e também entre $\mathrm{CH}$ e $\mathrm{CD}$;

$\beta$ é o ângulo de rotação do plano AGBF em torno de $\mathrm{AG}$, i.e., $\beta$ é o ângulo entre $\mathrm{AB}$ e $\mathrm{AC}$, que é igual ao ângulo entre $\mathrm{CD}$ e CA.

Figura 7. (a) Relações geométricas para o objeto (cordão de solda) e posição angular da câmera (Ponto A); (b) Construção geométrica auxiliar para a projeção do cordão w no plano perpendicular à chapa; (c) Construção geométrica auxiliar para projeção do cordão w no plano paralelo ao sensor da câmera.

Existem dois modelos a serem discutidos, cujas construções geométricas auxiliares são mostradas nas Figuras 7b e 7c. O primeiro modelo (Figura $7 b$ ) representa a projeção do cordão num plano perpendicular à chapa, ao passo que o segundo mostra a mesma projeção, mas num plano diferente e que seja paralelo ao sensor da câmera fotográfica. Ressalta-se que os

Vale ressaltar que o ângulo de rotação do plano ADHG em torno da linha $\mathrm{AB}$ não foi considerado. Este ângulo se refere à rotação do plano perpendicular ao objeto (cordão). Esta desconsideração foi adotada, pois pode ser facilmente compensada apenas rotacionando a imagem obtida ao final em qualquer programa comercial para tratamento de imagem.

Esta equação fornece a correção necessária a ser adotada quando se coloca a câmera numa determinada posição angular em relação ao objeto. Para demonstrar sua validade pode-se considerar que se $\alpha=0^{\circ}$ e $\beta=0^{\circ}$, então não se está observando qualquer imagem, ou seja, w' $=0$. Isto é provado 
matematicamente na Equação 7, fazendo inicialmente $\beta=0^{\circ}$, e após simplificando-a e fazendo $\alpha=0^{\circ}$. Desta forma, a Equação 7 fica demonstrada.

O objetivo da Equação 7 é calcular a relação entre a largura real do objeto (w) e sua projeção (w') medida na imagem. O ideal seria que não houve necessidade de qualquer correção, ou seja, w'=w. Isto pode ser obtido se a câmera for colocada numa posição angular tal que $\alpha=0^{\circ}$ e $\beta=45^{\circ}$. Isto comprova a sugestão inicial colocada no Item 2, para se colocar a câmera a $45^{\circ}$. Nesta opção não se faz necessário efetuar qualquer correção na medida retirada da imagem do ponto de vista geométrico do posicionamento angular do sistema de visão. Entretanto, deve-se atentar para calibrações em função das distorções que lentes e o próprio sensor da câmera apresentam.

Um ponto final a ser discutido refere-se ao correto posicionamento da câmera. Medições angulares não são triviais de serem obtidas com grande acuracidade. Assim, é interessante

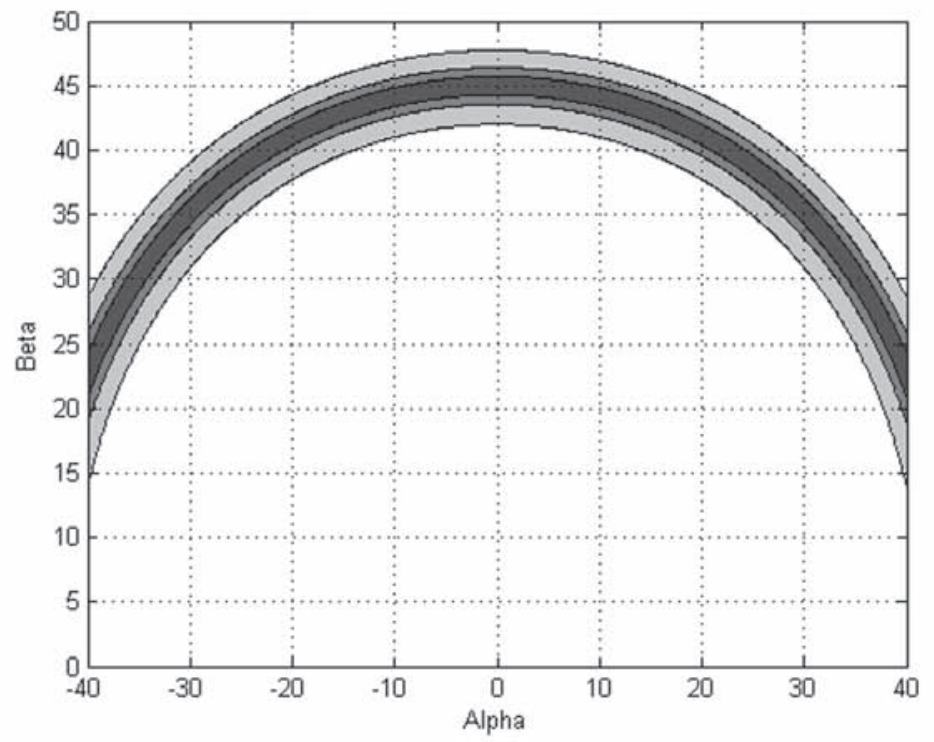

(a) definir o quanto pode-se afastar dos valores angulares onde não se requer correções nas medições realizadas na imagem. Além disto, pode haver restrições no posicionamento da câmera (acessibilidade de junta, por exemplo). Assim, é importante estabelecer o quanto se pode alterar os valores de $\alpha$ e $\beta$, para que não seja necessário efetuar correções na medição da imagem, dentro de uma margem de erro. Isto é conseguido pela Equação 7 e apresentado na Figura 8a para valores de erro em faixas de 2,$5 ; 5$ e $10 \%$ do valor correto obtido. Por esta figura, é possível estabelecer que se utilizando $\beta=45^{\circ}$, o ângulo $\alpha$ pode ser variado numa faixa de $-10^{\circ} \mathrm{a}+10^{\circ}$, mantendo-se o erro entorno de $\pm 2,5 \%$, o que é definitivamente importante para se obter uma maior visão angular sem que seja necessário efetuar correções ao longo da imagem (distorção maior na periferia do que no centro). Caso a variação possível do ângulo $\alpha$ seja muito estreita, deve-se aplicar diferentes correções ao longo de linhas verticais na imagem.

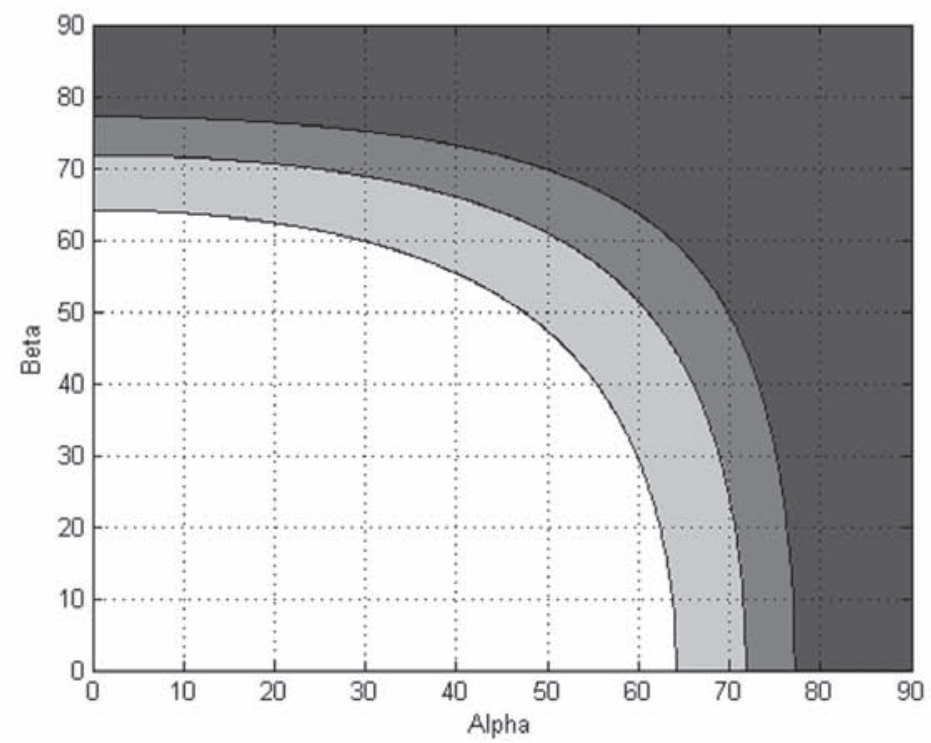

(b)

Figura 8. Valores possíveis para os ângulos e com base nas Equações 7(a) e 8(b) dentro de uma faixa de erro $\pm 2,5 \%$ (cinza escuro); $\pm 5 \%$ (cinza médio) e $\pm 10 \%$ (cinza claro), quando nenhuma compensação for feita na medição geométrica

Uma das considerações realizadas ao se estabelecer o modelo apresentado na Equação 7 é a projeção da imagem num plano perpendicular ao da peça. Outro modelo possível é considerar o plano da imagem perpendicular à linha de visão (Figura 7b), resultando na Equação 8.

$$
w_{p}^{\prime}=w \sqrt{1-\cos ^{2} \alpha \cdot \cos ^{2} \beta}
$$

onde, w' é a projeção da largura w do cordão no plano que contenha o sensor da câmera;

Semelhantemente como no modelo anterior, pode-se obter o gráfico da Figura 8b para valores de erro em faixas de 2,5; 5 e $10 \%$ do valor correto obtido. Por esta figura, fica claro que a única condição para se medir sem qualquer correção é obtida fazendo-se qualquer um dos ângulos igual a $90^{\circ}$, ou seja, paralelo à superfície da peça, o que é uma predição intuitiva.
Resta agora saber da aplicabilidade de cada modelo. O segundo modelo é aquele que mais se aproxima da realidade, pois contém o plano de projeção da imagem no sensor da câmera. Entretanto, a situação de projeção perpendicular é também presente, principalmente quando um grande campo de visão é observado, pois nem todos os objetos se encontram no mesmo plano. Assim, como o presente texto trata-se de um desafio, sugere-se aos leitores da Soldagem \& Inspeção que façam seus ensaios e observem qual modelo se aplica, discutam e verifiquem os limites de aplicação de cada um deles.

\subsection{Abertura do Diafragma}

Além da regulagem do 'shutter' e do uso de filtros, outra forma para se controlar a intensidade de luz que entra na câmera é através da abertura do diafragma da objetiva. O diafragma é composto por um conjunto de finas lâminas justapostas que 
se localiza dentro da objetiva e que permitem a regularem da intensidade de luz. A regulagem do diafragma se dá através de números, conhecidos como $\mathrm{F} /$ ponto ou número-F, que são dispostos em uma série numérica, cuja seqüência padrão é: $\mathrm{F} / 1,4 ; \mathrm{F} / 2 ; \mathrm{F} / 2,8 ; \mathrm{F} / 4 ; \mathrm{F} / 5,6 ; \mathrm{F} / 8 ; \mathrm{F} / 11 ; \mathrm{F} / 16 ; \mathrm{F} / 22$ e F/32, onde quanto menor for o número $\mathrm{f}$, maior a quantidade que luz que o diafragma permite passar. Cada número maior, ou seja, mais fechado, representa a metade da luz que a abertura anterior permite passar, assim como a cada número menor, ou seja, mais aberto, permite a entrada do dobro de luz. Esta característica deriva do fato de que os números-F indicam a divisão do raio médio da abertura do diafragma. Assim, por exemplo, usando uma abertura de F/8 entra metade da luz do que usando F/5,6 e o dobro de F/11. A atual escala de abertura utilizada pode ser obtida através da Equação 9.

$$
F=\frac{f}{A}
$$

\section{Onde,}

F é o número-F;

f é a distância focal e

A é o diâmetro da abertura do diafragma [mm].

A combinação da abertura com a velocidade de disparo ('shutter') não afeta apenas a quantidade de luz, mas também, o quanto da cena ficará nítido na foto final. A profundidade de campo, como é conhecida esta área, é a zona de nitidez aparente na frente e atrás do assunto em que a câmera está focalizando. $\mathrm{Na}$ prática, aberturas pequenas $(\mathrm{F} / 16, \mathrm{~F} / 22)$ geram profundidades de campo extensas, com quase tudo nítido, enquanto grandes aberturas $(F / 4, F / 2,8)$ fornecem áreas limitadas de nitidez, com apenas uma parte da cena em foco. Assim, diafragmas mais fechados tendem a proporcionar maior "foco", enquanto diafragmas mais abertos tendem a fazer o oposto, tendo em vista que ele aumenta ou diminui a profundidade de campo.

Em geral, recomenda-se sempre utilizar as aberturas medianas da objetiva que será utilizada, pois elas contêm a menor probabilidade de causar aberrações cromáticas e de problemas de refração nas bordas (quando utilizadas próximas a abertura máxima), ou de difração com o uso de aberturas muito pequenas.

Como exemplo, a Figura 9 traz a influência da variação do f/número no resultado fotográfico de um arco TIG, $100 \mathrm{~A}$, argônio puro. Do lado esquerdo desta figura, são apresentadas as imagens originais no padrão de cor RGB (Red-Green-Blue), enquanto que do lado direito é apresentada a mesma imagem, mas num padrão de cor diferente, denominado de HSV (HueSaturation-Value). Este padrão é interessante, pois realça as diferenças para o olho humano e facilita a visualização de contornos e tendências. Deve-se ressaltar que, do ponto de vista de informações nada se altera, ou seja, o comportamento pode ser obtido pela imagem RGB, com uso de processamento digital de imagem. Este assunto será discutido adiante. A imagem HSV é uma forma de superar as limitações do olho humano, quanto ao nível de contraste possível de serem observados a olho nu.

Por fim, apenas um lembrete que somente imagens 24 bits podem ser convertidas para HSV, haja vista que são necessários três canais com 8 bits cada.

\subsection{Canais de Cores}

Como citado no item anterior, é possível, através de processamento de imagens, extrair informações na forma de dimensões das imagens. Ao se tentar observar a variação da intensidade dos pixels de uma imagem ao longo da linha, a primeira dúvida que aparece é sobre qual canal usar: vermelho, verde ou azul (para uma imagem RGB). Poder-se-ia também converter a imagem para tons de cinza para se obter uma média dos canais.

Não existe uma resposta direta para este questionamento. Por exemplo, a Figura 10 traz um arco TIG e as diferentes abordagens que podem ser utilizadas para a mesma imagem. Neste caso, ressalta-se que o canal azul refere-se a comprimentos de onda menores (tendendo ao ultravioleta) e que são característicos de íons $\mathrm{Ar}^{+}$. Por espectroscopia óptica de emissão sabe-se que tais íons se concentram próximos ao eletrodo e ao se comparar as imagens para os diferentes canais, nota-se que as imagens para o canal azul possuem maior luminosidade na região próxima ao eletrodo. Raciocínio similar pode ser feito para o canal vermelho, que representa maiores comprimentos de onda, que são característicos de átomos excitados de argônio e estão presentes em regiões mais distantes do eletrodo, o que pode ser comprovado nas imagens.

\subsection{Calibração e Resolução}

Apesar da calibração de uma imagem parecer uma tarefa simples, ela não é tão direta quando se requer maior acuracidade das medidas. Tem havido uma tendência em se executar a calibração e medições de uma imagem, utilizando-se programas do tipo CAD. A calibração feita neste caso é somente uma calibração unidimensional, ou seja, a partir de uma dimensão conhecida da imagem. Esta forma de calibração não leva em conta distorções focais freqüentemente presentes na periferia de uma imagem. Também se assume que o objeto é perfeitamente plano, ou seja, se houve desníveis na imagem, estes não serão compensados. Por outro lado, existem softwares comerciais que utilizam até 8 posições para calibração a partir de uma grade fotografada/filmagem na mesma posição do objeto, proporcionando compensações para a maioria dos problemas relacionados.

A partir de uma dimensão conhecida na imagem, conseguese estabelecer a sua relação com o mundo real e definir a resolução da mesma em termos de escala real. De forma absoluta a resolução de uma imagem é definida multiplicandose o número de pixels horizontais pelos verticais. A escala da imagem em tamanho ( $\mathrm{mm} \times \mathrm{mm}$, por exemplo) está relacionada com a quantidade de pontos por polegada, ou dpi (dots per inch - pontos por polegada). Assim, uma imagem 100 x $100 \mathrm{~mm}$ com $72 \mathrm{dpi}$, pode ser ampliada para 100 x $100 \mathrm{~mm}$, mas terá 7,2 dpi, para a mesma resolução de 283 x 283 pixels.

A resolução absoluta de uma imagem pode ser definida pela sua menor medida, o pixel. Assim, a resolução da medida pode 

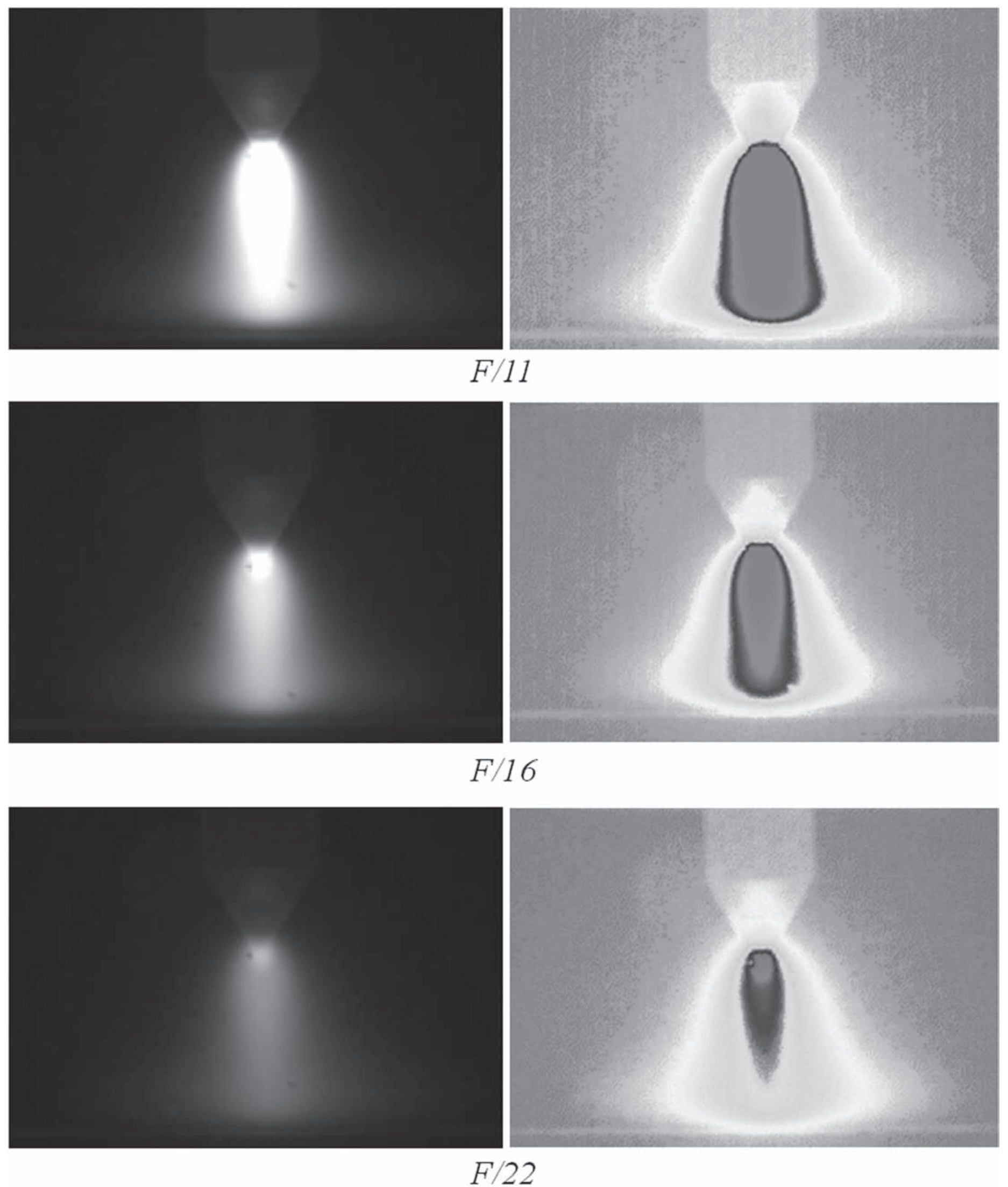

Figura 9. Resultado fotográfico da variação do f-número para um arco TIG, 100 A e argônio puro com eletrodo AWS WTh2 de 3,2 $\mathrm{mm}$ de diâmetro e comprimento do arco $5 \mathrm{~mm}$ sobre uma chapa de cobre resfriada [17]. Lado direito: imagens originais em RGB e do lado esquerdo: imagens em formato HSV. 


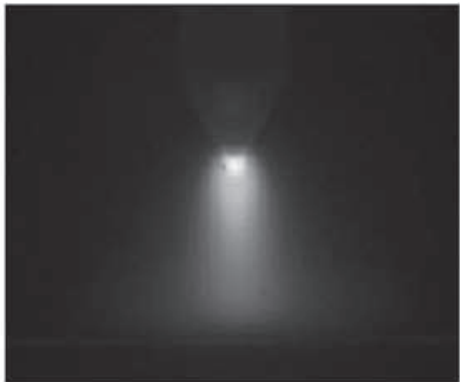

Imagem em $R G B$

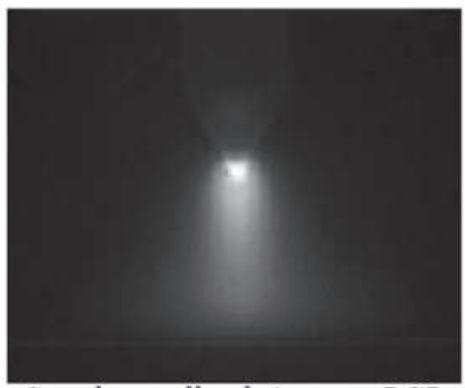

Canal vermelho da imagem $R G B$

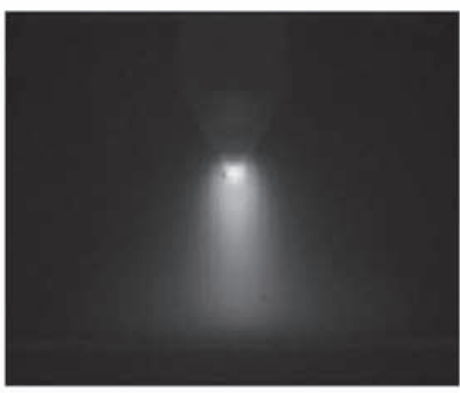

Canal verde da imagem $R G B$

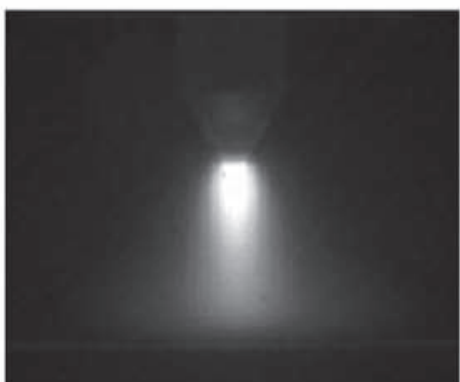

Canal azul da imagem $R G B$

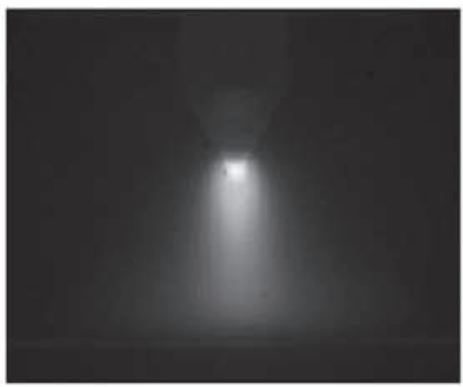

Imagem convertidapara tons de cinza

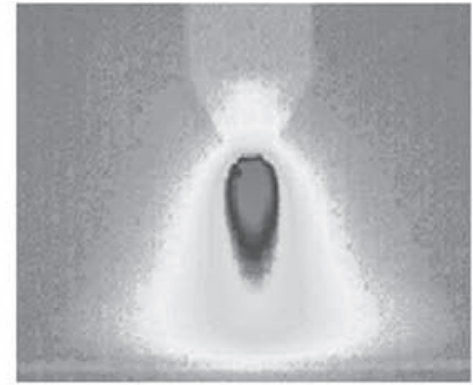

Imagem HSV a partir da imagem original

Imagem HSV para o canal vermelho

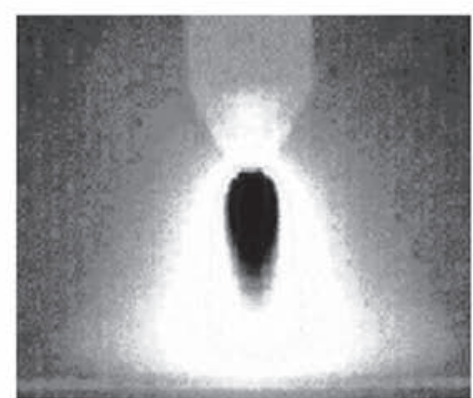

Imagem HSV para o canal verde

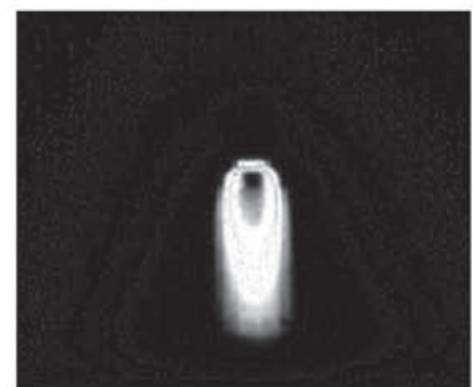

Imagem HSV para o canal azul

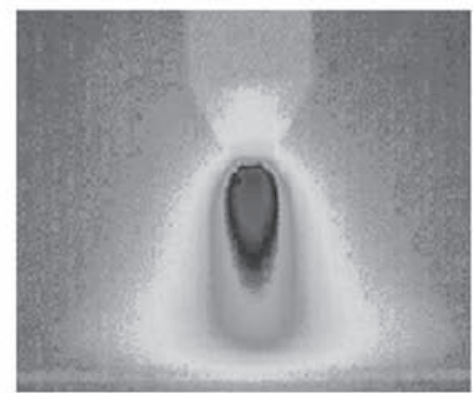

Imagem HSV para tons de cinza

Figura 10. Canais de cores para arco TIG, 70 A, argônio puro, F/16, comprimento de arco de 5 mm e eletrodo AWS WTh2 de 3,2 mm de diâmetro [17]. 
ser obtida dividindo-se a dimensão conhecida pelo número de pixels presentes nesta dimensão. Sugere-se utilizar dez vezes o valor obtido como variação mínima admissível ao se comparar medições das imagens.

\subsection{Tipos de Sensores}

De forma mais simples e prática, considerando os dois tipos principais de sensores utilizados comercialmente, o CCD (Charge Couple Device) e o CMOS (Complementary Metal Oxide), tem-se que a diferença na tecnologia se baseia em como a tensão de cada fotodiodo do sensor é lida. A tecnologia CCD é analógica, onde a tensão é convertida em digital através de um conversor analógico-digital (AD) ao longo da matriz do sensor em uma de suas quinas. No caso do CMOS, cada fotodiodo executa a conversão $\mathrm{AD}$, sendo denominado de pixel ativo, e o sinal já sai digital. Estas formas de trabalho definem as características de cada sensor. Sensores CCD são mais sensíveis a luz e produzem imagens de maior qualidade (menos ruído). Entretanto, são mais caros e consomem mais energia que os CMOS. Existe ainda, uma terceira tecnologia mais recente, o CMD (Charged-Modulated Devices), que é um híbrido entre CCD e CMOS. O CMD é analógico também, mas cada fotodiodo é tratado independentemente, como no CMOS. Câmeras de altavelocidade recentes fazem uso da tecnologia CMD.

Uma característica importante diz respeito à comparação entre imagens quando estas são obtidas em diferentes momentos. Por exemplo, um pesquisador gostaria de comparar a radiação (luz) emitida por um arco TIG e um arco Plasma através de fotografia. Neste caso, uma câmera que utilize sensor CMOS não pode ser utilizada, pois cada elemento do sensor é tratado de forma independente, ajustando-se em função da saturação da luz incidente, ou seja, cada imagem seria obtida a partir de uma função transferência diferente do sensor. Portanto, não se poderia compará-las. Para se efetuar tal comparação, o pesquisador deve utilizar uma câmera com sensor CCD, onde não existe este ajuste independente, uma vez que a matriz do sensor é tratada em conjunto.

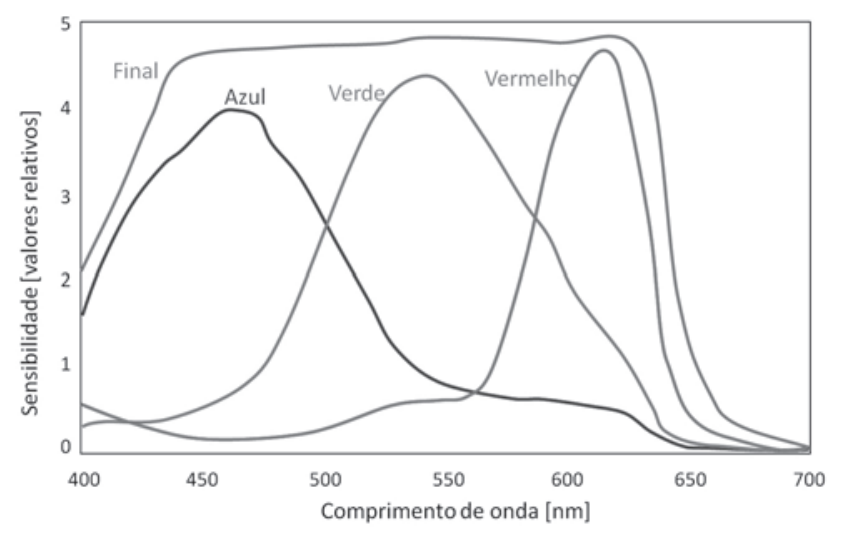

Figura 11. Exemplo ilustrativo de curva de sensibilidade de uma câmera CCD.
Um ponto final a ser destacado é com relação à curva de sensibilidade do sensor em função comprimento de onda da luz. Esta pode ser utilizada na escolha da câmera em função do objeto a ser visualizado e também na correção da imagem, caso seja necessário. As curvas de sensibilidade podem ser apresentadas para cada cor RGB ou de forma combinada. Um exemplo é apresentado na Figura 11.

\section{Observações Finais}

Espera-se a discussão aqui apresentada contribua para o melhor aproveitamento e correto uso das imagens/filmes obtidos em processos de soldagem. A técnica experimental de fotografia/filmagem é muito poderosa e quando corretamente utilizada mostra fenômenos importantes para o desenvolvimento tecnológico da soldagem.

Sem dúvida, as informações apresentadas podem ser utilizadas em qualquer área do conhecimento, mas os exemplos e abstrações foram dedicados à soldagem para melhor absorção da comunidade científica da área. Além disto, há ainda enormes potencialidades a serem exploradas como o uso de processamento de imagens para filtragem computacional das imagens.

Sugere-se que os leitores da Revista Soldagem \& Inspeção façam uma leitura cuidadosa e crítica do texto, apontando melhores interpretações, correções, sugestões e críticas para que os pesquisadores e usuários desta tecnologia possam mostrar imagens cada vez mais interessantes e corretas da soldagem.

\section{Agradecimentos}

O Prof. Vilarinho gostaria de agradecer à Capes, à Fapemig, ao CNPq e ao Laprosolda, além dos revisores pela observação detalhada do manuscrito inicial e importantes contribuições para a discussão do tema.

\section{Referências Bibliográficas}

[1] AWS/ANSI, ANSI Z49.1:1999 - Safety in Welding, Cutting, and Allied Processes. 1999. p. 61.

[2] NGUYEN, N. Thermal Analysis of Welds. Developments in Heat Transfer. Vol. 14, WIT Press, 2004. 334 p.

[3] VILARINHO, L.O., W. LUCAS, and S. RAGHUNATHAN. Governing Mechanisms in A-TIG Process. The Welding Institute - TWI, 2009, p. 170

[4] DONG, C., et al., Preliminary Study on the Mechanism of Arc Welding with the Activating Flux, in Aeronautical Manufacturing Technology, Supplement 2004. Paper presented at International Symposioum, High Energy Density Beam Processing Technology, Kunming, China, 12-17 Sept. 2004. p. 271-278.

[5] VARGAS, J.E., et al. Study of Resistance Spot Welding Process by Using Synchronized High Speed Filming. Soldagem \& Inspecao, v.11, p. 120-126, 2006.

[6] HOUGHTON, M.A., J.J. LUCAS, and W. LUCAS. Vision Systems for Monitoring and Control of Arc Welding Operations. Soldagem \& Inspecao, v.12, n. 4, p. 293-299, 2007. 
[7] RESENDE, A.A., F. KEOCHEGUERIANS, and L.O. VILARINHO. Influência dos Teores de $\mathrm{CO} 2$ e O2 na Corrente de Transição Globular-Goticular Utilizando Misturas a Base de Argônio Durante a Soldagem GMAW de Arame ER70S-6. Soldagem \& Inspecao, v.13, p. 50-57, 2009.

[8] SMITH, J.S. and E. AL. Vision Sensors in Arc Welding. IIW, Doc. XII-1850-05. 2005, p. 13

[9] FRANCO, L.D.N., Sinchronization, Capture and Analyses of Weld Pool Images in the GMAW Process in the Short-circuit Metal Transfer Mode (in Portuguese), in UnB. 2007, University of Brasilia: Brasilia. p. 143.

[10] IEC, Safety of laser products - Part 1: Equipment classification, requirements and user's guide - IEC 60825-1, Edition 1.2, 2001-08, IEC, Editor. 2001.

[11] VILARINHO, L.O., et al. Proposição de um Sistema de Aquisição e Controle em Soldagem via Transmissão de Dados sem Fio (Wireless). Universidade Federal de Uberlândia, 2007, p. 3

[12] HENRIKSON, P., Visualization of Weld Pool Surface Flow During TIG Welding on Stainless Steel 316L Plates, in Mathematical Modelling of Weld Phenomena 7, H.C.e. al, Editor. 2005. p. 125-147.

[13] OKAGAITO, T., F. MIYASAKA, and T. OHJI. UV Radiation Thermometry of TIG Weld Pool. IIW, Doc. 212-106104. 2004, p. 6

[14] ZHANG, U.M., H.S. SONG, and G. SAEED. Observation of a Dynamic Specular Weld Pool Surface. Meas. Sci. Technol., v.17, p. L9-L12, 2006.

[15] ZHANG, G., Z. YAN, and L. WU. Reconstructing a threedimensional P-GMAW weld pool shape from a two-dimensional visual image. Meas. Sci. Technol., v.17, p. 1877-1882, 2006.

[16] VILARINHO, L.O., Notas de Aula da Disciplina Instrumentação Aplicada à Manufatura. 2007, Universidade Federal de Uberlândia, Programa de Pós-Graduação em Eng. Mecânica. p. 3.

[17] VILARINHO, L.O. Characterisation of TIG Arc Structures Using Experimental Techniques. Cranfield University, 2002, p. 325 